\title{
Fostering Social Cognition through an Imitation- and Synchronization-Based Dance/Movement Intervention in Adults with Autism Spectrum Disorder: A Controlled Proof-of-Concept Study
}

\author{
Svenja Koehne ${ }^{a, b} \quad$ Andrea Behrends ${ }^{a, b} \quad$ Merle T. Fairhurst ${ }^{c} \quad$ Isabel Dziobek ${ }^{a, b}$ \\ ${ }^{a}$ Cluster of Excellence, Languages of Emotion, Freie Universität, and ${ }^{b}$ Berlin School of Mind and Brain, \\ Humboldt-Universität zu Berlin, Berlin, and 'Max-Planck Research Group, Early Social Development, \\ Max Planck Institute for Human Cognitive and Brain Sciences, Leipzig, Germany
}

\section{Key Words}

Autism · Social cognition - Empathy · Theory of mind . Imitation · Synchronization · Dance/movement intervention $\cdot$ Controlled trial

\begin{abstract}
Background: Since social cognition is impaired in individuals with autism spectrum disorder (ASD), this study aimed at establishing the efficacy of a newly developed imitation- and synchronization-based dance/movement intervention (SI$\mathrm{DMI}$ ) in fostering emotion inference and empathic feelings (emotional reaction to feelings of others) in adults with highfunctioning ASD. Methods: Fifty-five adults with ASD (IQ 285 ) who were blinded to the aim of the study were assigned to receive either 10 weeks of a dance/movement intervention focusing on interpersonal movement imitation and synchronization (SI-DMI, $\mathrm{n}=27$ ) or a control movement intervention ( $\mathrm{CMI}, \mathrm{n}=24$ ) focusing on individual motor coordination (2 participants from each group declined before baseline testing). The primary outcome measure was the objective Multifaceted Empathy Test targeting emotion inference and empathic feelings. Secondary outcomes were scores on the self-rated Interpersonal Reactivity Index. The well-established automatic imitation task and synchronization finger-tapping task were used to quantify effects on imitation and synchronization functions, complemented by
\end{abstract}

the more naturalistic Assessment of Spontaneous Interaction in Movement. Results: Intention-to-treat analyses revealed that from baseline to 3 months, patients treated with SI-DMI showed a significantly larger improvement in emotion inference $(d=0.58)$, but not empathic feelings, than those treated with $\mathrm{CMI}(\mathrm{d}=-0.04)$. On the close generalization level, SI-DMI increased synchronization skills and imitation tendencies, as well as whole-body imitation/synchronization and movement reciprocity/dialogue, compared to CMI. Conclusions: SI-DMI can be successful in promoting emotion inference in adults with ASD and warrants further investigation.

c) 2015 S. Karger AG, Basel

\section{Introduction}

Autism spectrum disorder (ASD) is characterized by impaired social interaction and communication and a restricted repertoire of activities and interests [1]. Deficits in social cognition, e.g., inferring others' mental states such as emotions and perspective taking, are at the core of the disorder [2-4]. Socioemotional functions such as empath-

Merle T. Fairhurst is now at the Center for the Study of the Senses, School of Advanced Study, University of London, UK.

\section{KARGER}

E-Mail karger@karger.com

www.karger.com/pps
(C) 2015 S. Karger AG, Basel

0033-3190/15/0851-0027\$39.50/0
Svenja Koehne

Berlin School of Mind and Brain

Humboldt-Universität zu Berlin, Unter den Linden 6

DE-10099 Berlin (Germany)

E-Mail svenja.koehne@hu-berlin.de 
ic feelings (being emotionally affected by someone else's emotions) have also been suggested to be aberrant, though less consistently [5-8]. Rigorously tested treatment options for adults with ASD targeting social cognition deficits, however, are scarce, as shown by the fact that only 3 randomized controlled trials were undertaken, with very small sample sizes [9]. Of note, those interventions target compensatory mechanisms focusing on explicit top-down learning strategies. Although individuals with ASD have been shown to profit from explicit strategies, they are likely to demand cognitive resources and thus add strain in real-life social situations [10]. Consequently, it may alternatively be promising to develop interventions for social cognition that are implicit in nature.

In social interaction humans show spontaneous coordination of movements, i.e. imitation of facial expressions and postures and movement synchronization such as falling into lock-step when walking side by side [for review, see 11]. Interestingly, both imitation and synchronization have been reported aberrant in autism [12$14]$ and it has been suggested that those dysfunctions contribute to social cognition deficits $[15,16]$. For instance, Marsh et al. [12] showed that children with ASD tend to synchronize their rocking-chair movements less with those of their parents compared to typically developing children. Here we suggest that interpersonal movement synchronization and imitation might be a target for social-cognitive interventions in ASD.

Our rationale is backed up by extensive basic research linking imitation and synchronization to social cognition [17]. It has been shown that synchronizing movements with those of another person during an interaction is associated with greater rapport, feelings of closeness and pro-social behavior [18-23]. Similarly, imitation of postures, facial expressions, and other behaviors entail positive social consequences including liking, emotion recognition, generosity, and reduced racial prejudice [24-27]. In addition, the modulation of spontaneous imitation through top-down control (imitation inhibition) has been suggested to play a crucial role in perspective taking [28-30].

Interestingly, the idea of using imitation and synchronization in clinical interventions to target social functions has a long tradition in dance/movement therapy in general and in working with children with autism in particular [31-34]. Dance/movement therapists apply an empathic reflection of their client's movements (mirroring of the movement quality and/or its form) to build a relationship and enhance emotional understanding between the therapist and the client [35]. A recent 7-week intervention study focusing on mirroring in movement showed improved self-reported social skills in young adults with autism [36], providing some empirical evidence for this notion.

Taken together, fostering interpersonal movement imitation and synchronization might serve as an effective leverage to enhance social cognition in adults with ASD, but experimental evidence of its efficacy is lacking. In the context of this study we developed an imitation- and synchronization-based dance/movement intervention (SI-DMI) for adults with ASD aiming at fostering social cognition. We aim at showing that SI-DMI enhances sociocognitive (emotion inference and perspective taking) and socioaffective processes (empathic feelings) in adults with ASD compared to a control movement intervention (CMI).

\section{Methods}

This participant-blinded, controlled parallel-group trial took place at the Psychology Department of Freie Universität Berlin, Germany, from December 2011 to March 2013 and was approved by the ethics committee of the German Psychological Society (DGP).

\section{Participants}

Eligible participants $(n=55)$ were aged 18 through 55 years with a diagnosis of high-functioning ASD, average intelligence (IQ $>85$ ), right-handedness without severe physical conditions, and not currently undergoing psychotherapy. ASD participants were recruited through the autism outpatient clinic of the Charité University Medicine Berlin or referred to us by specialized clinicians and centers and were diagnosed according to DSM-IV/ICD10 criteria for Asperger disorder and autistic disorder using the Autism Diagnostic Observation Schedule (ADOS) [37] and the Autism Diagnostic Interview - Revised (ADI-R) [38] if parental informants were available. Participants gave written informed consent.

\section{Interventions}

SI-DMI and CMI were administered in ten 90-min sessions over the course of 3 months in small groups of 4-10 members (group size varied based on participant's availability and due to dropouts). Participants were blinded to the goal of the study and fully debriefed in writing at the end. To provide a rationale for participating in the intervention, both SI-DMI and CMI were announced as movement programs for adults with ASD to promote motor functions and general physical well-being.

SI-DMI was developed and manualized jointly by a child and adolescent psychiatrist with dance movement therapy education (A.B.) and a professional dancer/choreographer. Compared to integrative dance movement therapy, SI-DMI realized a more focused and standardized approach that has been theoretically outlined in further detail elsewhere [17]. A full description of SI-DMI is in preparation by A.B. Each session of SI-DMI followed the same structure (table 1). The primary components (70\%) were exercises for dyads, small groups, and the entire group focusing on interpersonal movement imitation and synchronization with and without music. For instance, in mirroring tasks participants imi- 
Table 1. Session structure of SI-DMI

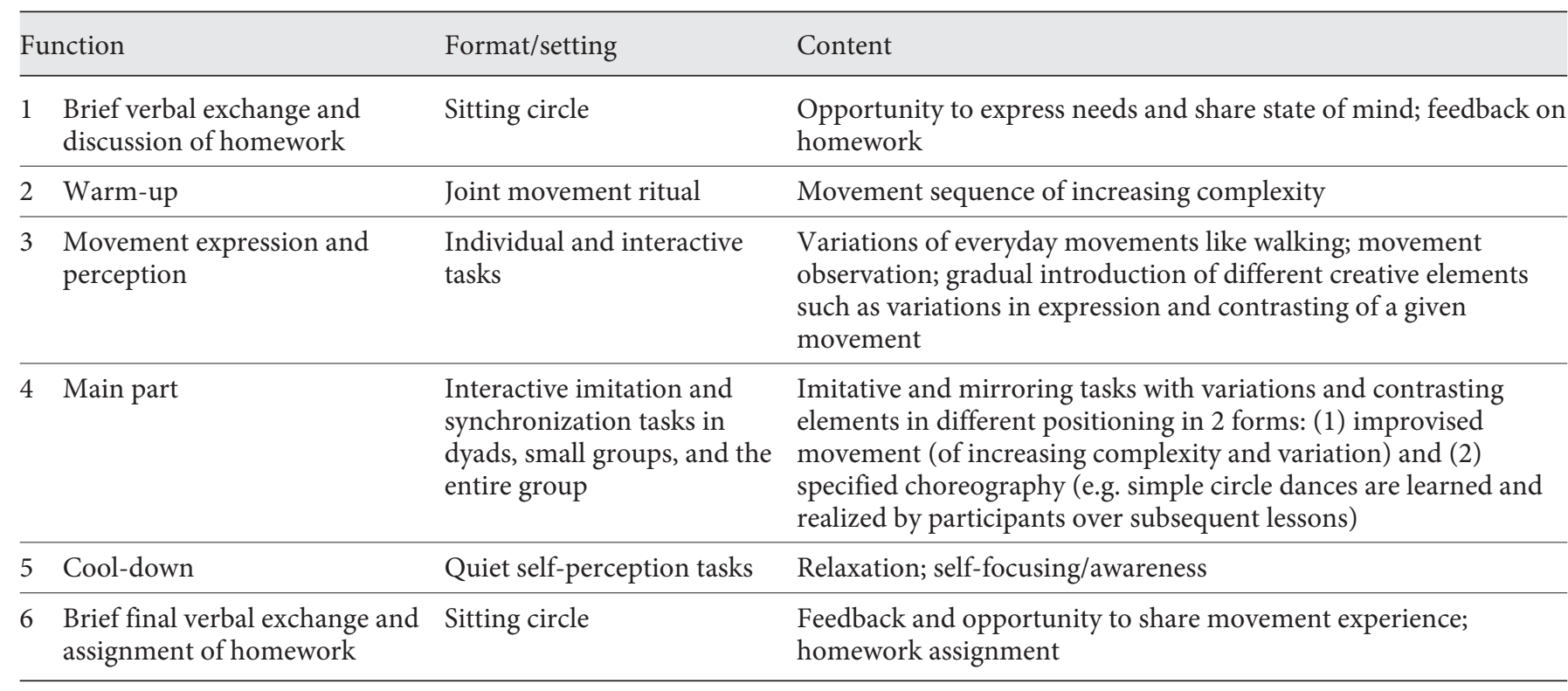

tated a repetitive movement initiated by another participant (such as arm swings) until synchrony occurred. In circle dances, participants learned choreographed movement sequences through imitation over the course of several sessions and then performed the dance in synchrony. Movement complexity and improvisation of movement gradually increased over the course of the intervention. Since top-down modulation of the spontaneous tendency to imitate (imitation inhibition) has been shown to affect perspective taking [28-30], exact imitation/synchronization tasks were alternated with tasks focusing on variation and turn taking in joint movement in every session. For example, during a mirroring task, participants were asked to vary the size and speed of a movement that they had previously imitated. The most advanced form of variation and turn taking was a face-to-face movement dialogue in which participants built on each other's movements to create interaction in movement. In order to strengthen the perception of their own body and individual movement expression as basic requirements for reciprocity in movement [17], respective tasks were also integrated in every session (approx. 30\%). Homework such as observing and imitating movements in others was assigned to facilitate the transfer of movement observation and imitation into every-day life interactions. The structure and contents of the sessions were specifically adapted to the needs of individuals with autism, which entailed among others predictable session structure, avoidance of sensory overload (e.g. interpersonal connection through ropes instead of direct touch), and possibility for time out.

Although CMI was also administered in a group setting, it did not involve tasks on interpersonal movement imitation and synchronization, but individual tasks on dexterity, balance, and endurance (e.g. juggling, simple yoga poses, or practicing on exercise machines) that were offered in a circle training-like format. All tasks were offered in 3-4 difficulty levels and participants monitored their progress in diaries to enhance motivation. While each task was introduced and shown briefly at the beginning of each

Dance/Movement Intervention for ASD session, detailed instructions were provided in writing to avoid learning through imitation. In addition, no two participants performed the same task at the same time and all faced the wall to minimize observation of movements while performing an exercise. Physical effort and structural aspects such as group setting, session structure, use of music, verbal exchange, and homework were kept constant across interventions.

\section{Outcome Measures}

On a close generalization level we expected SI-DMI to enhance imitation and synchronization, which would on a distant generalization level transfer into improved emotion inference and empathic feelings. Thus, the Multifaceted Empathy Test (MET) [39] was our primary outcome and the Interpersonal Reactivity Index (IRI) [40] together with imitation/synchronization paradigms and the more naturalistic Assessment of Spontaneous Interaction in Movement (ASIM) our secondary outcome measures, which were assessed at baseline and within 4 weeks after the intervention.

Primary Outcome Measure. The MET [39] is an ecologically valid measure that allows for the simultaneous assessment of emotion inference and empathic feelings and has been proven sensitive in ASD. The computer-based MET consists of 40 photographs depicting people in emotionally charged situations. To assess emotion inference, subjects are required to infer the emotions of the individuals shown in the photographs by selecting one of four mental state descriptors. To assess empathic feelings, subjects rate how much they share the feelings of the individuals displayed in the pictures on a 9-point Likert scale. The MET has been shown to be a reliable measure of emotion inference (Cronbach's alpha $=$ $0.71)$ and empathic feelings (alpha $=0.91)$ [39].

\section{Secondary Outcome Measures}

Interpersonal Reactivity Index

Individual differences in self-rated perspective taking and empathic concern on the trait level were assessed using the IRI [40]. 
The perspective taking scale assesses the tendency to spontaneously adopt the psychological point of view of others (e.g. 'I try to understand my friends better by imagining how things look from their perspective'). The empathic concern scale taps the respondent's feelings of warmth, compassion, and concern for others (e.g. 'I often have tender, concerned feelings for people less fortunate than me').

\section{Automatic Imitation}

To measure imitation tendencies we used the automatic imitation paradigm, which has been well established in basic research [41]. In summary, participants perform a cued finger-lifting task while seeing task-irrelevant videos of either of the following: (1) a hand simultaneously performing the same movement in a mirrored fashion or (2) a static hand. Reaction time differences between conditions (1) and (2) were used as a proxy for automatic imitation, assuming that the tendency of automatic imitation leads to shorter reaction times in the moving hand condition(see online suppl. Appendix for a detailed description and data from incongruent condition; see www. karger.com/doi/10.1159/000441111 for all online suppl. material).

\section{Interpersonal Synchronization}

A finger-tapping task was used that has previously been developed to investigate dynamic human interactions as they occur during group music making and dance [42]. Participants were asked to tap in synchrony with the tones of virtual drumming partners, who were programmed to be either human-like (variably adaptive to the participant's performance) or mechanic like a metronome (nonadaptive; online suppl. Appendix, fig. 1). The standard deviation (SD) of asynchronies between taps and tones served as a measure for synchronization performance, with higher values reflecting weaker performance.

\section{Assessment of Spontaneous Interaction in Movement}

The ASIM is an observation coding schedule allowing for a systematic and standardized assessment of dyadic interactional whole-body movements and forms part of a new standardized assessment of a person's individual and interactional movement profile which we developed specifically for this study because no other naturalistic assessment of spontaneous, interactional wholebody movement was available [43]. The assessment is based on the film-based movement profile analysis [44], which was designed to describe movement quality in the course of dance/ movement therapy. Participants were asked to engage in simple 1- to 1.5-min movement and dance tasks to music, e.g. walking, in a predefined space. Two tasks (expanding/contracting and swing movements) were then repeated while a confederate joined the movement space. There was no instruction to interact, allowing for spontaneous interaction in movement for the participant. The confederate followed a semi-standardized choreography, which included several nonverbal offers to interact with the participant (e.g. turning toward the participant). Video recordings of the interactive movement set were analyzed for four spontaneous interactive movement criteria: (1) orientation of gaze and body towards confederate, (2) relation in spatial movement, (3) imitation/synchronization and (4) reciprocity/dialogue in movement. The ASIM coding criteria comprise quantitative and qualitative movement aspects. All movement parameters were judged on a scale ranging from 0 (not shown) to 3 (clearly and frequently shown, flexible and socially modulated) with detailed descriptions for each score. An example of the coding scheme is given in online supplementary table 1. Ratings for the four different interactional movement patterns reflect an average of the two interactive movement tasks. Ratings were done by one of the authors (A.B.), who was not blinded to group allocation. To evaluate the reliability of this measure, 5 baseline assessments (2 individuals from CMI group and 3 from SI-DMI) and 3 additional assessments from neurotypical individuals were picked at random and rated by a second trained rater who was blinded to the diagnoses as well as group allocation. Two-way mixed, single-measure (consistency agreement) intraclass correlations (ICC) were in the excellent range for spontaneous imitation/synchronization ( ICC $=0.92$ ), reciprocity/dialogue (ICC $=0.78$ ), and relation in spatial movement $($ ICC $=0.85)$. The ICC was only fair for the gaze and body orientation criterion ( ICC $=0.45)$. Overall, the results indicate that raters had a high degree of agreement and suggest that the ASIM is a reliable measure of more qualitative aspects of spontaneous movement in interaction.

\section{Group Allocation}

Between December 2011 and March 2013 patients were invited to participate in a movement program advertised to target general motor functions and well-being. All group sessions (SI-DMI and CMI groups) were offered at different times of the week and with varying start dates throughout the year. After a telephone screening targeting exclusion criteria, participants (who were all blinded to the purpose of the study) chose their group based on time preferences. Participants were allocated sequentially in order of their declaration of their preference and a group was closed after including 10 participants. Each participant was allocated prior to baseline testing and first personal contact to guarantee that allocation could not be influenced based on symptom severity, level of functioning, or baseline results.

\section{Statistical Analysis}

Sample size calculations were based on previous studies on the effect of experimental manipulations of imitation/synchronization on social cognition $[18,19,27,45]$ reporting medium-to-large effect sizes $(\mathrm{d}=0.36-1.07)$. Thus, for our pre-post design, we proposed a conservative medium effect size of $\eta_{\mathrm{p}}{ }^{2}=0.13$, resulting in a total sample size of $\mathrm{n}=56$, given a significance level of $5 \%$ and power of $80 \%$. Thus, all 55 individuals evaluated as eligible were included in the study. Analyses were conducted on the modified intention-to-treat (ITT) population with all participants analyzed in their assigned treatment condition. For participants who did not start $(n=2)$ or complete $(n=1)$ the intervention, data were imputed using the last observation carried forward. Alternative methods for handling missing data - namely complete case analysis and multiple imputations - yielded very similar results and levels of significance (online suppl. tables 2,3). Outcome measures were analyzed using a repeated-measure mixed-model ANOVA with the intersubject factor of time (before and after intervention) and the between-subject factor of group (SI-DMI and CMI). Post hoc $t$ tests to further investigate interaction effects were Bonferroni corrected. Effect sizes for the interaction effects were calculated using $\eta_{\mathrm{p}}{ }^{2}$. For an estimation of clinical significance, Cohen's $\mathrm{d}$ was calculated for the treatment effect (difference between before and after intervention) for both interventions separately. All analyses were 2-tailed with a p value threshold of $5 \%$ and run using SPSS (version 20.0). 


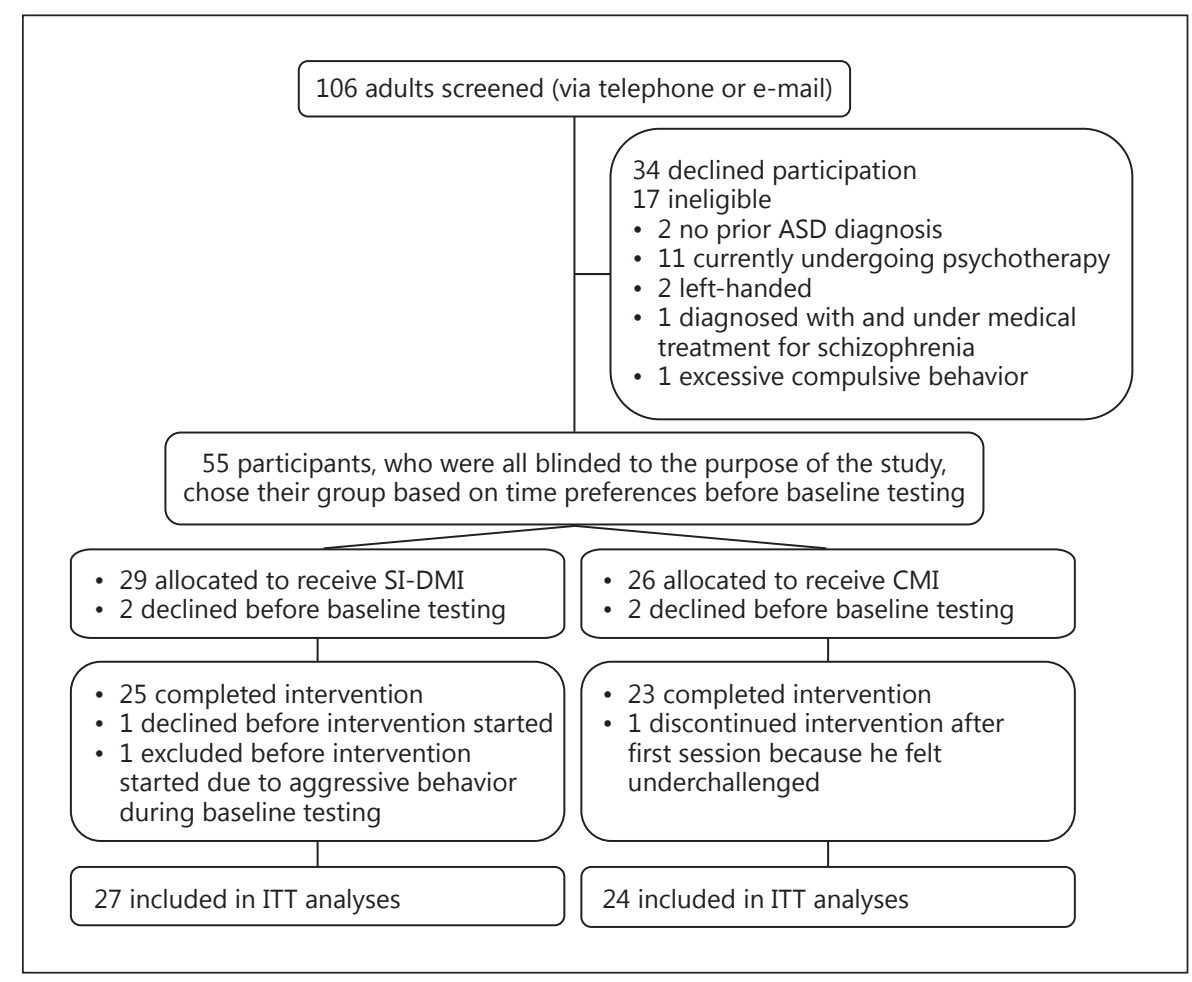

Fig. 1. Flow of patients through the trial.

\section{Missing Data}

One subject from the CMI group was excluded from the automatic imitation analyses because he showed prolonged reaction times ( $>3$ SD from group mean) across all conditions at postintervention assessment. Only a subset of participants (SI-DMI: $\mathrm{n}=16$, CMI: $n=9$ ) underwent ASIM due to restricted personal resources. All participants tested with the ASIM before intervention were also tested after intervention and included in the analyses. All other missing data points are due to technical problems during data acquisition.

\section{Results}

\section{Recruitment}

Between December 2011 and March 2013, 106 adults with high-functioning ASD were screened and those 55 evaluated as eligible were assigned to one of the two treatment conditions (fig. 1). Four participants declined before baseline testing; 51 were included in ITT analyses. There were no significant between-group differences in terms of gender, age, intelligence (measured by a vocabulary test, Wortschatztest, WST) [46], and symptom severity [47] (table 2). From those 51 participants included, additional parental information (ADI-R) was available for 16 participants. Most participants missed no sessions and only 2 participants (SI-DMI group) missed a 3rd session. Missed sessions were substituted by extra homework.

\section{Outcomes}

Details of all ITT analyses are shown in table 3. Improvements in emotion inference but not empathic feelings as measured with the MET were bigger in the SIDMI group than in the CMI group $\left(\mathrm{p}=0.04, \eta_{\mathrm{p}}{ }^{2}=0.09\right)$. Post hoc t tests confirmed that emotion inference was significantly increased after SI-DMI $(\mathrm{p}=0.008)$ but not after CMI ( $p=0.65$ ). Cohen's $d$ for the difference between means at baseline and after treatment was $d=0.58$ for the SI-DMI group compared to $\mathrm{d}=-0.04$ for the CMI group, thus confirming the clinical significance of the improvement in emotion inference [48]. There were no significant changes in self-ratings of trait-level perspective taking (IRI; $\mathrm{p}=0.68$ ) or empathic concern (IRI; $\mathrm{p}=0.71$ ).

On the close generalization level, a significant interaction effect of group $\times$ time revealed that the automatic imitation effect was increased in the SI-DMI group compared to the CMI group $\left(\mathrm{p}=0.04, \eta_{\mathrm{p}}{ }^{2}=0.08\right)$ after intervention. Post hoc $t$ tests confirmed that automatic imitation was significantly increased after SI-DMI $(\mathrm{p}=0.02$, $\mathrm{d}=0.47)$ but not after CMI ( $\mathrm{p}=0.51, \mathrm{~d}=-0.03)$. Simi- 
Table 2. Baseline demographic and clinical characteristics

\begin{tabular}{lccc}
\hline Characteristic & SI-DMI (n=27) & CMI (n=24) & p value \\
\hline Male sex & 18 & 14 & 0.54 \\
Age, years & $33.5(9.1)$ & $32.0(9.1)$ & 0.54 \\
In a relationship or married & $3(11.11)$ & $3(12.5)$ & 0.88 \\
Finished high school ${ }^{1}$ or vocational training & $20(74.07)$ & $17(70.83)$ & 0.80 \\
Crystalline intelligence, IQ (WST) & $106.7(12.9)$ & $109.8(9.1)$ & 0.39 \\
Symptom severity (ADOS) & $10.3(5.2)$ & $10.6(4.9)$ & 0.80 \\
Autism Spectrum Quotient (AQ) & $36.7(7.3)$ & $36.0(10.2)$ & 0.79 \\
Depressive symptoms (BDI-II) & $16.4(12.3)$ & $14.0(11.0)$ & 0.46 \\
BSI Global Severity Index & $0.91(0.57)$ & $0.81(0.61)$ & 0.54 \\
Received psychotherapy in the 6 months before baseline & $5(18.52)$ & $5(20.83)$ & 0.84 \\
$\quad$ Behavioral therapy & 3 & 1 & 2 \\
Psychoanalysis or psychodynamic therapy & 1 & 2 & \\
Other/not specified & 1 & & \\
\hline
\end{tabular}

Data are presented as means (SD) or $\mathrm{n}(\%) . \mathrm{WST}=$ Wortschatztest (German vocabulary test); ADOS = Autism Observation Schedule; AQ = Autism Quotient; BDI-II = Beck Depression Inventory [55]; BSI = Brief Symptom Inventory [56]. $\mathrm{p}$ values for continuous variables were generated from independent sample $\mathrm{t}$ tests; $\mathrm{p}$ values for categorical variables were generated from Pearson's $\chi^{2}$ tests.

${ }^{1}$ High school: German equivalent of high school degree after 13 years of schooling.

Table 3. Outcome measures at baseline and after intervention

\begin{tabular}{|c|c|c|c|c|c|c|c|c|c|c|c|c|c|}
\hline Outcome & \multicolumn{5}{|c|}{ SI-DMI } & \multicolumn{5}{|c|}{ CMI } & $\begin{array}{l}\mathrm{F} \\
\text { value }\end{array}$ & $\begin{array}{l}\mathrm{p} \\
\text { value }\end{array}$ & $\begin{array}{l}\text { Effect } \\
\text { size, } \\
\eta_{\mathrm{p}}{ }^{2}\end{array}$ \\
\hline Empathic feelings (MET) & 27 & $\begin{array}{l}3.51 \\
(2.0)\end{array}$ & $\begin{array}{l}3.64 \\
(2.0)\end{array}$ & $\begin{array}{l}0.13 \\
(-0.2 \text { to } 0.4)\end{array}$ & 0.15 & 24 & $\begin{array}{l}3.80 \\
(2.1)\end{array}$ & $\begin{array}{l}3.85 \\
(1.9)\end{array}$ & $\begin{array}{l}-0.05 \\
(-0.4 \text { to } 0.3)\end{array}$ & -0.06 & 0.64 & 0.43 & 0.01 \\
\hline Empathic concern (IRI) & 27 & $\begin{array}{l}13.44 \\
(5.7)\end{array}$ & $\begin{array}{l}13.74 \\
(5.3)\end{array}$ & $\begin{array}{l}0.30 \\
(-1.2 \text { to } 1.8)\end{array}$ & 0.08 & 24 & $\begin{array}{l}14.67 \\
(7.0)\end{array}$ & $\begin{array}{l}15.38 \\
(5.5)\end{array}$ & $\begin{array}{l}0.71 \\
(-0.9 \text { to } 2.3)\end{array}$ & 0.18 & 0.14 & 0.71 & 0.003 \\
\hline Automatic imitation effect ${ }^{2}$ & 27 & $\begin{array}{l}20.42 \\
(40.1)\end{array}$ & $\begin{array}{l}37.88 \\
(34.5)\end{array}$ & $\begin{array}{l}17.46 \\
(2.4 \text { to } 32.5)\end{array}$ & 0.47 & 23 & $\begin{array}{l}42.55 \\
(37.5)\end{array}$ & $\begin{array}{l}37.13 \\
(32.9)\end{array}$ & $\begin{array}{l}-5.42 \\
(-21.8 \text { to } 10.9)\end{array}$ & -0.03 & 4.29 & 0.04 & 0.08 \\
\hline $\begin{array}{l}\text { Asynchrony }{ }^{3} \text { with human-like } \\
\text { (adaptive) virtual partner }\end{array}$ & 19 & $\begin{array}{l}26.80 \\
(6.5)\end{array}$ & $\begin{array}{l}24.47 \\
(4.7)\end{array}$ & $\begin{array}{l}-2.32 \\
(-3.9 \text { to }-0.7)\end{array}$ & -0.63 & 15 & $\begin{array}{l}28.56 \\
(4.7)\end{array}$ & $\begin{array}{l}28.95 \\
(4.3)\end{array}$ & $\begin{array}{l}0.36 \\
(-1.4 \text { to } 2.1)\end{array}$ & 0.13 & 5.39 & 0.03 & 0.14 \\
\hline Gaze and body orientation (ASIM) & 16 & $\begin{array}{l}1.69 \\
(1.8)\end{array}$ & $\begin{array}{l}1.88 \\
(1.9)\end{array}$ & $\begin{array}{l}0.18 \\
(-0.5 \text { to } 0.1)\end{array}$ & 0.27 & 9 & $\begin{array}{l}1.44 \\
(0.8)\end{array}$ & $\begin{array}{l}1.44 \\
(1.0)\end{array}$ & $\begin{array}{l}0.0 \\
(-0.4 \text { to } 0.4)\end{array}$ & 0 & 0.49 & 0.49 & 0.02 \\
\hline $\begin{array}{l}\text { Relation in spatial movement } \\
\text { (ASIM) }\end{array}$ & 16 & $\begin{array}{l}1.16 \\
(2.0)\end{array}$ & $\begin{array}{l}1.56 \\
(2.1)\end{array}$ & $\begin{array}{l}0.41 \\
(0.0 \text { to } 0.9)\end{array}$ & 0.43 & 9 & $\begin{array}{l}0.94 \\
(0.8)\end{array}$ & $\begin{array}{l}0.83 \\
(0.9)\end{array}$ & $\begin{array}{l}-0.11 \\
(-0.7 \text { to } 0.5)\end{array}$ & -0.47 & 2.09 & 0.16 & 0.08 \\
\hline
\end{tabular}

Baseline and postintervention data are means (SD) from ITT analyses (carry forward). CI = Confidence interval; MET = Multifaceted Empathy Test; IRI = Interpersonal Reactivity Inventory; ASIM = Assessment of Spontaneous Interaction in Movement. p values and effect sizes $\left(\eta_{p}{ }^{2}\right)$ refer to time $\times$ group interactions from mixed model ANOVAs. ${ }^{1}$ Cohen's d, effect size for the change from baseline to after intervention within each group. ${ }^{2}$ Difference between reaction times in the compatible and baseline conditions in milliseconds. ${ }^{3} \mathrm{SD}$ of asynchronies between the participant's tap and their virtual drumming partner's tone in milliseconds; higher values reflect poorer performance. 
larly, asynchrony while interacting with human-like (variably adaptive) partners in the finger-tapping task was reduced after SI-DMI compared to CMI $(p=0.03$, $\left.\eta_{\mathrm{p}}{ }^{2}=0.14\right)$. Again, post hoc $\mathrm{t}$ tests showed that asynchrony decreased significantly in the SI-DMI group $(\mathrm{p}=0.005$, $\mathrm{d}=-0.63)$ but not in the CMI group $(\mathrm{p}=0.68, \mathrm{~d}=0.13)$. Interestingly, asynchronies were unaffected by the interventions when interacting with a mechanic (nonadaptive) partner (all $\mathrm{p}>0.51$ ).

Results of the ASIM revealed that SI-DMI increased the quantity and quality of spontaneous imitation and synchronization of movements $\left(\mathrm{p}=0.001, \eta_{\mathrm{p}}^{2}=0.4\right)$, as well as reciprocity/dialogue in movement $(\mathrm{p}=0.009$, $\eta_{\mathrm{p}}^{2}=0.26$ ), compared to CMI. Both interaction effects were driven by a significant increase of imitation/synchronization $(\mathrm{p}<0.001, \mathrm{~d}=1.27)$ and reciprocity/dialogue $(\mathrm{p}=0.04, \mathrm{~d}=1.25)$, respectively, in the SI-DMI group, whereas both parameters did not change significantly in the CMI group (imitation/synchronization: $\mathrm{p}=$ $0.08, \mathrm{~d}=-0.47$; reciprocity/dialogue: $\mathrm{p}=0.59, \mathrm{~d}=-0.16$ ) In contrast, orientation of gaze and body towards the confederate and relation in spatial movement were not differentially affected by the two interventions (orientation: $\mathrm{p}=0.49, \eta_{\mathrm{p}}{ }^{2}=0.02$; relation in spatial movement: $\mathrm{p}=0.16$, $\left.\eta_{\mathrm{p}}^{2}=0.08\right)$.

\section{Discussion}

A dance/movement intervention based on interpersonal movement imitation and synchronization (SIDMI) was effective in fostering emotion inference in adults with high-functioning ASD compared to a CMI focusing on individual movement tasks. On a close generalization level, the SI-DMI group showed increased imitation tendencies and enhanced synchronization abilities compared to the CMI group.

In the primary outcome measure, the MET, the SIDMI group showed an increase in emotion inference but not in empathic feelings compared to the CMI group. The effect of SI-DMI on emotion inference is in line with previous research, relating imitation, top-down modulation of imitation (imitation inhibition), and interpersonal movement synchronization to sociocognitive processes including emotion recognition and mentalizing $[27,28,42$, $49,50]$. In contrast to our study, previous research has also suggested that imitation and synchronization increase socioaffective processes such as liking and affiliation [for review, see 11]. An explanation for the null effect on empathic feelings found here could be that imitating and moving in synchrony with other individuals influence affective states such as liking, affiliation, and empathic feeling in the situation, i.e. on the state level, directed specifically towards the individuals that were interacted with. In contrast, a skill such as emotion inference refers to a learned process that can be applied to other situations more easily. This might explain why, in the post-treatment assessment, changes could only be observed in emotion inference but not empathic feelings. No changes in perspective taking or empathic concern were measured with the IRI. Questionnaires, however, demand high introspection [51], which has been reportedly reduced in ASD [52]. Future studies should include follow-up assessments to ascertain whether effects extend to subjectively perceived changes in social cognition as time passes.

On the close generalization level we found an increase in the tendency to automatically imitate movements in the SI-DMI group but not in the control group. In addition, the ability to synchronize movements was increased in the SI-DMI group compared to controls when interacting with a virtual human-like partner but not when interacting with a nonadaptive mechanic virtual partner, which further underlines the social nature of the changes observed in sensorimotor functions. These changes were corroborated by the ecologically more valid ASIM assessment, showing that also on the whole-body level, imitation and synchronization as well as movement reciprocity and dialogue increased in the SI-DMI group compared to the CMI group. In sum, these effects show that SI-DMI indeed enhanced interpersonal imitation and synchronization. Importantly, they also suggest that an increase of quantity and quality of imitation/synchronization might underlie the observed increase in emotion inference.

Existing social interventions for adults with ASD such as 'Mindreading' [53] or 'PEERS for young adults' [54] mostly focus on teaching the understanding of others' minds, e.g. emotions, explicitly, they rely heavily on compensatory strategies and intellectual functions [53] and explicit rule-based learning $[9,53,54]$. Those interventions are thus of limited applicability for individuals with ASD and intellectual disability. SI-DMI, in contrast, focuses on synchronization and imitation of movements, which do not demand explicit cognitive strategies, thereby reducing the cognitive strain in social situations. Thus, although only adults with high-functioning ASD were included in the present study, we believe that individuals with low intellectual functioning might benefit from the intervention in an adapted form as well. Further research is needed to confirm the appropriateness and effectiveness of SI-DMI in cognitively more impaired individuals with ASD. 
By targeting social cognitive functions directly most interventions remain agnostic with respect to associated mechanisms that might be promising in mitigating respective dysfunctions in ASD. SI-DMI focuses on imitation and synchronization as likely precursors of emotion inference and empathic feelings. In showing that our intervention indeed generalizes beyond motor functions to emotion inference we might speculate about mechanisms capable of enhancing social deficits more generally. Basic research points to at least 3 potential mechanisms that may have contributed to the observed effects of SI-DMI: imitation, top-down modulation of imitation (imitation inhibition), and interpersonal movement synchronization [11, 28]. Since all 3 aspects were combined in SI-DMI, this study does not allow disentangling whether the observed effects were due to one of these mechanisms or their combination. Future research should focus on linking specific interpersonal motor patterns to specific social-cognitive functions to allow for more targeted dance/movement interventions to be developed that mitigate social dysfunctions. For instance, evidence is particularly strong for a link between synchronization and socioaffective consequences $[18,23]$. In contrast, imitation and imitation inhibition have been specifically linked to sociocognitive functions, i.e., emotion recognition and perspective taking, respectively $[27$, $28,30,49]$, which should be explored in more detail.

Our study is limited by the focus on one measure of emotion inference and restriction to high-functioning adults with ASD. Since results were partly marginal and not controlled for multiple comparisons they need replication, especially with longer follow-up periods, to verify the efficacy of SI-DMI before it can be recommended for clinical use. In addition, while we found it important to complement our objective, computer-based tests of imitation and synchronization within a more ecologically valid and qualitative measure, results from the ASIM are limited because the rater was not blinded to group allocation.
This being said, our study also has several strengths, as we kept a number of important aspects constant between SIDMI and the control intervention such as the setting, structure of the sessions, use of music, and homework assignments. Unlike other studies, we did not rely solely on self-report measures in individuals with ASD but also on objective and possibly more valid computer-based measures of social functions and imitation/synchronization, complemented by more naturalistic movement analyses. In addition, our sample was larger than most comparable studies and all participants were blinded.

Taken together, we found that a dance/movement intervention focusing on interpersonal imitation and synchronization increased emotion inference beyond enhancing imitation and synchronization functions. This suggests that interpersonal motor coordination functions might be a promising means towards treating core social cognitive deficits in ASD, which warrants further investigation.

\section{Acknowledgments}

This study was funded by the German Research Foundation (DFG; grant No. EXC302). Merle T. Fairhurst was supported by a grant from the Arts and Humanities Research Council (grant No. AH/L007053/1).

We thank the following individuals for their uncompensated support of our study: Nicola Strehle and Shu Zhang (support of control group implementation) and Stefan Haydn and Katharina Bartels (data collection). We furthermore thank the following individuals for their compensated participation in this study: Sybille Müller, Claudia Dietrich, and Natascha Roy (assistance in design of movement interventions, and administration, of movement interventions) and Lilly Schmidt and Sebastian Siegler (administrative support, data collection, data management, and analysis).

\section{Disclosure Statement}

The authors report no financial or other relationship relevant for this article.

\section{References}

1 American Psychiatric Association: Diagnostic and Statistical Manual of Mental Disorders, ed 5. Washington, American Psychiatric Association, 2013.

-2 Harms MB, Martin A, Wallace GL: Facial emotion recognition in autism spectrum disorders: a review of behavioral and neuroimaging studies. Neuropsychol Rev 2010;20:290322

3 Baron-Cohen S: Theory of mind and autism : a review. Int Rev Res Ment Retard 2001;23: 169.
4 Schwenck C, Mergenthaler J, Keller K, Zech J, Salehi S, Taurines R, et al: Empathy in children with autism and conduct disorder: group-specific profiles and developmental aspects. J Child Psychol Psychiatry 2012;53: 651-659.

5 Bird G, Silani G, Brindley R, White S, Frith U, Singer T: Empathic brain responses in insula are modulated by levels of alexithymia but not autism. Brain 2010;133:1515-1525.
6 Rogers K, Dziobek I, Hassenstab J, Wolf OT, Convit A: Who cares? Revisiting empathy in Asperger syndrome. J Autism Dev Disord 2007;37:709-715.

7 Hadjikhani N, Zürcher NR, Rogier O, Hippolyte L, Lemonnier E, Ruest T, et al: Emotional contagion for pain is intact in autism spectrum disorders. Transl Psychiatry 2014; 4:e343.

8 Minio-Paluello I, Baron-Cohen S, Avenanti A, Walsh V, Aglioti SM: Absence of embodied empathy during pain observation in Asperger syndrome. Biol Psychiatry 2009;65:55-62. 
-9 Bishop-Fitzpatrick L, Minshew NJ, Eack SM: A systematic review of psychosocial interventions for adults with autism spectrum disorders. J Autism Dev Disord 2013;43:687-694.

10 Glaser R: The reemergence of learning theory within instructional research. Am Psychol 1990;45:29-39.

11 Chartrand TL, Lakin JL: The antecedents and consequences of human behavioral mimicry. Annu Rev Psychol 2013;64:285-308.

-12 Marsh KL, Isenhower RW, Richardson MJ, Helt M, Verbalis AD, Schmidt RC, et al: Autism and social disconnection in interpersonal rocking. Front Integr Neurosci 2013;7:4.

13 Vanvuchelen M, Roeyers H, De Weerdt W: Do imitation problems reflect a core characteristic in autism? Evidence from a literature review. Res Autism Spectr Disord 2011;5:8995.

-14 Edwards LA: A meta-analysis of imitation abilities in individuals with autism spectrum disorders. Autism Res 2014;7:363-380.

15 Perkins T, Stokes M, McGillivray J, Bittar R: Mirror neuron dysfunction in autism spectrum disorders. J Clin Neurosci 2010;17: 1239-1243.

16 Kana RK, Wadsworth HM, Travers BG: A systems level analysis of the mirror neuron hypothesis and imitation impairments in autism spectrum disorders. Neurosci Biobehav Rev 2010;35:894-902.

17 Behrends A, Müller S, Dziobek I: Moving in and out of synchrony: a concept for a new intervention fostering empathy through interactional movement and dance. Arts Psychother 2012;39:107-116.

18 Wiltermuth SS, Heath C: Synchrony and cooperation. Psychol Sci 2009;20:1-5.

19 Hove MJ, Risen JL: It's all in the timing: interpersonal synchrony increases affiliation. Soc Cogn 2009;27:949-960.

20 Bernieri FJ: Coordinated movement and rapport in teacher-student interactions. J Nonverbal Behav 1988;12:120-138.

21 Kirschner S, Tomasello M: Joint music making promotes prosocial behavior. Evol Hum Behav 2010;31:354-364.

22 Cacioppo S, Zhou H, Monteleone G, Majka EA, Quinn KA, Ball AB, et al: You are in sync with me: neural correlates of interpersonal synchrony with a partner. Neuroscience 2014; 277:842-858.

23 Catmur C, Heyes C: Is it what you do, or when you do it? The roles of contingency and similarity in pro-social effects of imitation. Cogn Sci 2013;37:1541-1552.

24 Bate S, Cook SJ, Mole J, Cole J: First report of generalized face processing difficulties in Möbius sequence. PLoS One 2013;8.

-25 Van Baaren RB, Holland RW, Steenaert B, van Knippenberg A: Mimicry for money: behavioral consequences of imitation. J Exp Soc Psychol 2003;39:393-398.

26 Dimberg U, Thunberg M, Elmehed K: Unconscious facial reactions to emotional facial expressions. Psychol Sci 2000;11:86-89.
7 Stel M, van Knippenberg A: The role of facial mimicry in the recognition of affect. Psychol Sci 2008;19:984-985.

28 Brass M, Ruby P, Spengler S: Inhibition of imitative behaviour and social cognition. Philos Trans R Soc Lond B Biol Sci 2009;364:2359_ 2367.

29 Santiesteban I, Banissy MJ, Catmur C, Bird G: Enhancing social ability by stimulating right temporoparietal junction. Curr Biol 2012;22: 2274-2277.

30 Santiesteban I, White S, Cook J, Gilbert SJ, Heyes C, Bird G: Training social cognition: from imitation to theory of mind. Cognition 2012;122:228-235.

31 Adler J: Looking for me. Berkeley, Berkeley Extension, 1970.

32 Samaritter R, Payne H: Kinaesthetic intersubjectivity: a dance-informed contribution to self-other relatedness and shared experience in non-verbal psychotherapy with an example from autism. Arts Psychother 2013;40:143150.

33 Archambeau MK, Szymanski DJ: Dance therapy and the autistic child. J Psychol Exp Res 1977;9:54-55

34 Scharoun SM, Reinders NJ, Bryden PJ, Fletcher PC: Dance/movement therapy as an intervention for children with autism spectrum disorders. Am J Dance Ther 2014;36:209228.

35 Sandel SL: The process of empathic reflection in dance therapy; in Sandel SL, Chaiklin S, Lohn A (eds): Foundations of Dance/Movement Therapy. The Life and Work of Marian Chace. Columbia, Marian Chace Memorial Fund of the American Dance Therapy Association, 1952, pp 98-111.

36 Koch SC, Mehl L, Sobanski E, Sieber M, Fuchs T: Fixing the mirrors: a feasibility study of the effects of dance movement therapy on young adults with autism spectrum disorder. Autism 2015;19:338-50.

37 Lord C, Rutter M, DiLavore PC, Risi S: Autism Diagnostic Observation Schedule: ADOS. Los Angeles, Western Psychological Services, 2002.

38 Lord C, Rutter M, Le Couteur A: Autism Diagnostic Interview - Revised: a revised version of a diagnostic interview for caregivers of individuals with possible pervasive developmental disorders. J Autism Dev Disord 1994; 24:659-685.

39 Dziobek I, Rogers K, Fleck S, Bahnemann M, Heekeren HR, Wolf OT, et al: Dissociation of cognitive and emotional empathy in adults with Asperger syndrome using the Multifaceted Empathy Test (MET). J Autism Dev Disord 2008;38:464-473.

40 Davis MH: Measuring individual differences in empathy: evidence for a multidimensional approach. J Pers Soc Psychol 1983;44:113126.

41 Cook J, Bird G: Atypical social modulation of imitation in autism spectrum conditions. J Autism Dev Disord 2012;42:1045-1051.
42 Fairhurst MT, Janata P, Keller PE: Being and feeling in sync with an adaptive virtual partner: brain mechanisms underlying dynamic cooperativity. Cereb Cortex 2013;23:25922600.

43 Behrends A, Dziobek I: Assessment of Spontaneous Interaction in Movement (ASIM). In preparation.

44 Bräuninger I, Züger B: Filmbasierte Bewegungsanalyse zur Behandlungsevaluation von Tanz- und Bewegungstherapie (film-based movement analysis in treatment evaluation of dance movement therapy); in Koch SC, Bender S (eds): Movement Analysis - Bewegungsanalyse. The legacy of Laban, Bartenieff, Lamb and Kestenberg. Berlin, Logos, 2007, pp 213-223.

45 Valdesolo P, Ouyang J, DeSteno D: The rhythm of joint action: synchrony promotes cooperative ability. J Exp Soc Psychol 2010; 46:693-695.

46 Schmitz K-H, Metzler P: Wortschatztest: WST. Weinheim, Beltz, 1992.

47 Baron-Cohen S, Wheelwright S, Skinner R, Martin J, Clubley E: The autism-spectrum quotient $(\mathrm{AQ})$ : evidence from Asperger syndrome/high-functioning autism, males and females, scientists and mathematicians. J Autism Dev Disord 2001;31:5-17.

48 Samsa G, Edelman D, Rothman ML, Williams GR, Lipscomb J, Matchar D: Determining clinically important differences in health status measures. Pharmacoeconomics 1999;15: 141-155.

49 Oberman LM, Winkielman P, Ramachandran VS: Face to face: blocking facial mimicry can selectively impair recognition of emotional expressions. Soc Neurosci 2007;2:167178 .

50 Yun K, Watanabe K, Shimojo S: Interpersonal body and neural synchronization as a marker of implicit social interaction. Sci Rep 2012;2:959.

51 Scheier MF, Carver CS: Self-focused attention and the experience of emotion: attraction, repulsion, elation, and depression. J Pers Soc Psychol 1977;35:635-636.

52 Happé F: Theory of mind and the self. Ann NY Acad Sci 2003;1001:134-144.

53 Golan O, Baron-Cohen S: Systemizing empathy: teaching adults with Asperger syndrome or high-functioning autism to recognize complex emotions using interactive multimedia. Dev Psychopathol 2006;18:591-617.

54 Gantman A, Kapp SK, Orenski K, Laugeson EA: Social skills training for young adults with high-functioning autism spectrum disorders: a randomized controlled pilot study. J Autism Dev Disord 2012;42:1094-1103.

55 Beck A, Steer R, Brown G: Manual for the Beck Depression Inventory - II, 1996.

56 Derogatis LR: BSI Brief Symptom Inventory: Administration, Scoring, and Procedure Manual, ed 4. Minneapolis, National Computer Systems, 1993. 